\title{
Introduction to the RE'10 special issue Requirements Engineering in a multi-faceted World
}

\author{
Jane Cleland-Huang
}

Published online: 7 August 2011

(C) Springer-Verlag London Limited 2011

The 18th International Requirements Engineering Conference, held in Sydney, Australia, from September 27, 2010 to October 1, 2010, addressed the challenges of "Requirements Engineering in a multi-faceted World." This theme highlighted the rich and diverse contexts of software and systems engineering projects, where requirements engineering methodologies, tools, and processes, are influenced by factors such as the size, domain, location, criticality, people, and organizational culture of a project.

The origins of the requirements engineering conference series stems back to the IEEE Symposium on Requirements Engineering held in San Diego, California, in 1993. For 8 years, the symposium alternated with the Conference on Requirements Engineering, until the two series were merged to form a single conference in 2002. The current merged conference provides an annual forum for researchers and practitioners to engage in a lively discussion and debate of current issues and challenges related to both the theory and practice of requirements engineering.

The 18th International Conference on Requirements Engineering hosted three excellent keynote speakers. Dr. Al Davis, Professor at the University of Colorado at Colorado Springs, challenged participants to keep their eyes focused on the end goal of their research and to deliver solutions that ultimately impact and improve the state of practice. Dr. Mary O'Kane, Chief Scientist and Scientific Engineer in the State of New South Wales, Australia, and also Executive Chairman of a Sydney-based company that advises governments, universities, and the private sector on innovation, research, education, and development, contextualized requirements engineering issues from the perspective of

J. Cleland-Huang ( $\square)$

School of Computing, DePaul University, Chicago, IL, USA

e-mail: jhuang@cs.depaul.edu
Australia's recent growth in both government and industrial sectors. Finally, Dr. Graeme Simsion, who served as CEO of a business and information systems consultancy company and now works as an independent consultant, shared his perspectives on consulting and ethics in the requirements engineering domain.

The 18th International Requirements Engineering Conference included both an industry and a research track which fostered lively and insightful conversations between academics and practitioners. The research track received 153 viable submissions. A multi-tiered review process was followed in which each paper was evaluated by three program committee members, and papers with at least one positive review were discussed further by program committee and board members. Ultimately 24 full-length papers were accepted, and three short papers, producing an acceptance rate of just under 16\%. Program Committee members were then asked to recommend papers for consideration for this special edition of the Requirements Engineering Journal. Eight papers were recommended and ultimately five of them were selected for submission. All of these papers have undergone a standard journal review process with subsequent revisions, and we are pleased to present them in this special issue of REJ.

The first paper accepted to this special issue of REJ, entitled "Analysing anaphoric ambiguity in natural language requirements" by Yang, de Roeck, Gervasi, Willis, and Nuseibeh, investigates the problem of nocuous ambiguities. These occur when requirements are written in natural language, and humans disagree on their interpretation. This paper, which won the best paper award at the conference, describes an automated approach for identifying such ambiguities in a requirements specification. Highlighting potential problem areas allows humans to discuss and disambiguate the impacted requirements. 
The second selected paper "Utilizing Event-B for domain engineering: a critical analysis," by Mashkoor and Jacquot, describes the experiences of the authors in using the formal framework of Event-B to model the safetycritical domain of a transportation system. The authors provide an insightful analysis into the use of Event-B on a real project and highlight both its strengths and weaknesses. This paper builds on a long history of work in formal specifications at the Requirements Engineering Conference.

The third selected paper "Application of Swarm techniques to requirements tracing" by Sultanov, Huffman Hayes, and Kong, uses swarm intelligence techniques, taken from the Artificial Intelligence community, to generate traceability matrices between high- and low-level requirements. This work addresses the ongoing challenge of automating the creation and maintenance of requirements traceability links and contributes to the growing body of knowledge in this area.

The fourth paper, "Representing and Reasoning about Preferences in Requirements Engineering" by Liaskos, McIlraith, Sohrabi, and Mylopoulos, extends existing goal modeling notations to integrate the concept of requirements priorities. The new model quantifies priorities so that they can be used to evaluate alternate design options.

Last, but not least, the fifth paper "Relevance-based abstraction identification: technique and evaluation" by Gacitua, Sawyer, and Gervasi, presents a technique for discovering abstractions from descriptions of the problem domain. This approach is designed to reduce the cognitive load of the requirements engineer during the domain analysis process.

I would also like to acknowledge the outstanding work of the 10 year impact award recipients. Unlike best papers which are selected purely on the quality and potential of the work, the impact award recognizes work that has had a significant influence on both research and practice over the past decade. This year's award recognizes the work of Carl
Gunter, Else Gunter, Michael Jackson, and Pamela Zave for their 2,000 paper from the International Symposium on Requirements Engineering, entitled "A Reference Model for Requirements and Specifications." This paper defined a reference model for applying formal methods to the development of user requirements and their reduction to a system's behavioral specification. One of the primary contributions of this work is the formal expression of a requirements engineering problem which has since engaged the interest of numerous researchers and has, according to Michael Jackson, in his impact award acceptance letter, "developed into a discipline of problemoriented software engineering that has proved its worth in practical application." This paper has therefore not only influenced future research but has also made a significant impact on the long-term practice of software engineering.

It is my privilege as Program Chair of RE'10, to organize this special issue of the REJ journal; however, this would not have been possible without the dedication of many people who worked so hard to make the conference a success. I would especially like to thank Didar Zowghi, General Chair of RE'10, who hosted the conference at the University of Technology, Sydney. I would also like to thank Neil Maiden, the current chair of the RE Conference Steering Committee, as well as Peri Loucopoulus, Bill Robinson, and the REJ editorial team for their ongoing support. Finally, I would like to thank members of the RE'10 organizing committee, program committee, and program board, as well as additional anonymous reviewers who reviewed the papers for this special edition of REJ.

My hope is that this special edition will provide interesting insights into the specific problems addressed by the five selected papers and will encourage new researchers to engage in requirements engineering research. To this end, I invite you to participate in future Requirements Engineering conferences to be held in Trento, Italy in 2011, and Chicago, USA, in 2012. 